\title{
Führung virtueller Teams
}

Eine gute Führungskraft zeichnet sich durch den souveränen und den konstruktiven Umgang mit gesellschaftlichen und unternehmerischen Herausforderungen sowie ein hohes Maß an Selbstreflexion aus. Dennoch nehmen die Anforderungen an die Führung zahlenmäßig und an Komplexität konstant zu. Dies kann einen Manager im digitalen Zeitalter schnell überfordern. Die Abb. 5.1 zeigt nur einen Ausschnitt der zahlreichen Herausforderungen, mit denen sich Führungskräfte konfrontiert sehen. Dazu zählt die Führung virtueller Teams, für die neben neuen Vorgehensweisen auch ein hohes Maß an Selbstreflexion erforderlich ist. Selbst Bundeskanzlerin Angela Merkel reagiert aktuell (04/2020) aufgrund von COVID-19 das Land aus dem Homeoffice und steht vor der gleichen Herausforderung (vgl. Zeit 2020c).

In Kap. 4 wurden Informationen und Empfehlungen zu Meetingformaten und Arbeitsmethoden gegeben, die dabei helfen können, ein virtuelles Team zu organisieren. Wie Sie diese als Führungskraft sinnvoll in Ihre operative und tägliche Führung integrieren, um virtuellen Teams zu Höchstleistungen zu verhelfen und Ihre Mitarbeiter aus dem Homeoffice zielführend zu steuern, soll im vorliegenden Kapitel behandelt werden. Die Empfehlungen werden nach den Aspekten persönliche Ebene (Charakter und Verhalten) und technologische Ebene (Führung mit Technologie) unterteilt.

\subsection{Persönliche Ebene: Ein neuer Typus Manager?}

Sicherlich haben Sie schon viel über Führung gelesen. Seit den Veröffentlichungen von Peter Drucker in den 80er Jahren wechselt die Diskussion des Führungsstil von Kommando und Kontrolle auf eine menschliche und 


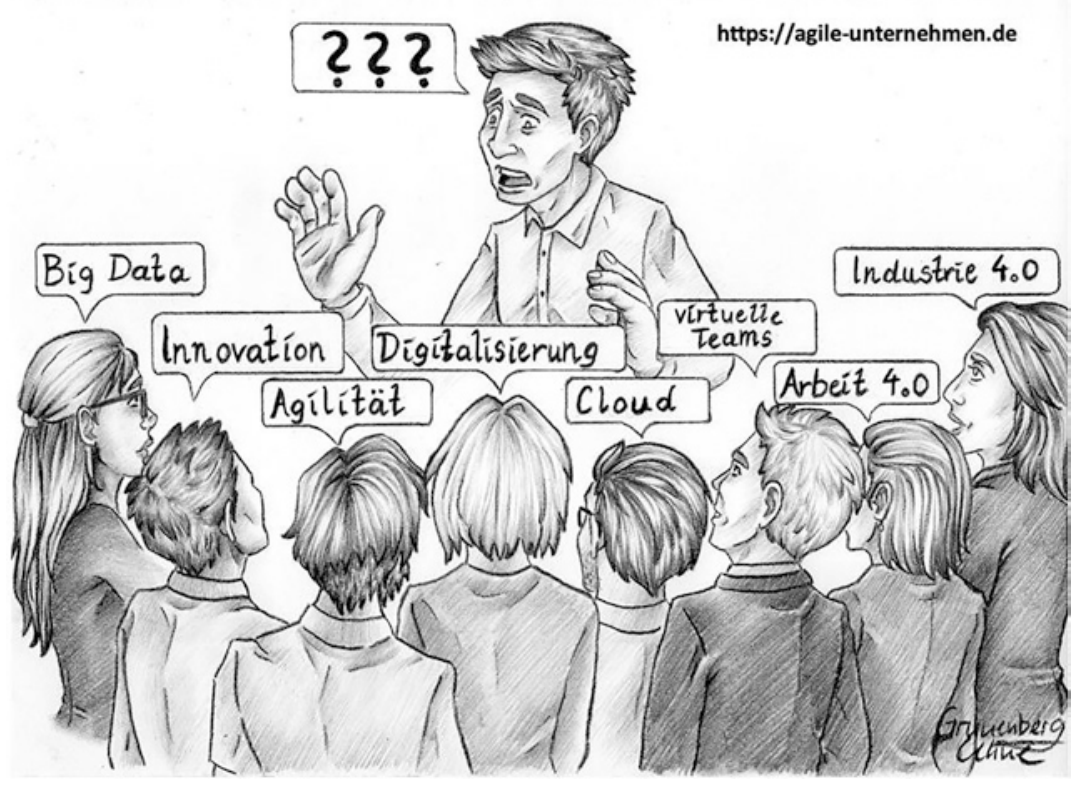

Abb. 5.1 Führungskräfte haben es im digitalen Zeitalter nicht leicht

authentische Führung (vgl. Drucker 2014). Diese beiden Aspekte waren, wie mir scheint, lange Zeit eher weniger bedeutsam und wurden erst in den letzten fünf Jahren durch die Medien in den Vordergrund gerückt. So wurde beispielsweise der oft leger gekleidete und in der Sprache sehr direkte Fußballtrainer Jürgen Klopp vom Manager Magazin für ,Management mit Charisma, Chuzpe (Dreistigkeit) und Cleverness“ (Zitat: Manager Magazin 2019) als Manager des Jahres gewählt. Diese Entscheidung steht im deutlichen Gegensatz zu jenen der Vorjahre dar und löste eine kontroverse Diskussion aus. Ein weiteres Beispiel ist Ex-Daimler-Vorstand Dieter Zetsche, der die Krawatte bei Daimler abschaffte und bei einer Präsentation mit einem T-Shirt mit der Aufschrift ,Do-Epic-Shit * für einen regelrechten Skandal in den Top-Management-Etagen deutscher Dax-Konzerne sorgte (vgl. Wirtschaftswoche 2018).

Beide Manager stellten einen Antityp zum alten, grauhaarigen Mann mit Krawatte und Anzug dar und brechen mit dem klassischen Managerbild, das sich seit den 1980er Jahren in den Köpfen etabliert hat. In diesem Zusammenhang scheint mit jungen Startup-Gründern wie Daniel Kraus (Flixbus), Valentin 
Stalf (N26), Dominik Richter (HelloFresh) und Anna Alex (Outfittery) eine neue Generation an Managern mit Charakter, Authentizität, Chuzpe und legerer Kleidung zu entstehen.

\subsection{Empfehlungen zur Führung auf persönlicher Ebene}

In meiner Forschungsarbeit zur Digitalisierung von Unternehmen wird gute Führung als eine, Verhaltensweise“ oder eine ,persönliche Einstellung ' einer Führungskraft beschrieben (vgl. Lindner und Greff 2018). Doch wie kann eine solche authentische Führung von einer Führungskraft umgesetzt werden?

Ich habe dazu gemeinsam mit Tobias Greff (vgl. Lindner und Greff 2019) über 65 Führungskräfte $\mathrm{zu}$ einem Leitbild befragt. Im Ergebnis wurden acht Werte festgehalten, die die Führungskräfte als besonders wichtig hervorgehoben haben. Die Führungskräfte sollten diese Werte gegeneinander gewichten und die Schieberegler so einstellen, dass ein persönliches Wertebild entsteht. Dieses finden Sie in der Abb. 5.2.

Merkbox Stellen Sie sich die Frage: Wie möchte ich sein und wie möchte ich wahrgenommen werden?

Die Grundidee besteht darin, weder komplett agil noch , unagil` zu sein, sondern eine Balance zwischen den sogenannten alten und den neuen Werten zu finden, sozusagen wie ein DJ an einem Mischpult, an dem gewisse Einstellungen wie Höhen oder Bass vorgenommen werden können. Neben dem Wertebild lassen sich folgende Empfehlungen der befragten Führungskräfte zusammenfassen (vgl. Lindner 2019b sowie Lindner und Greff 2019):

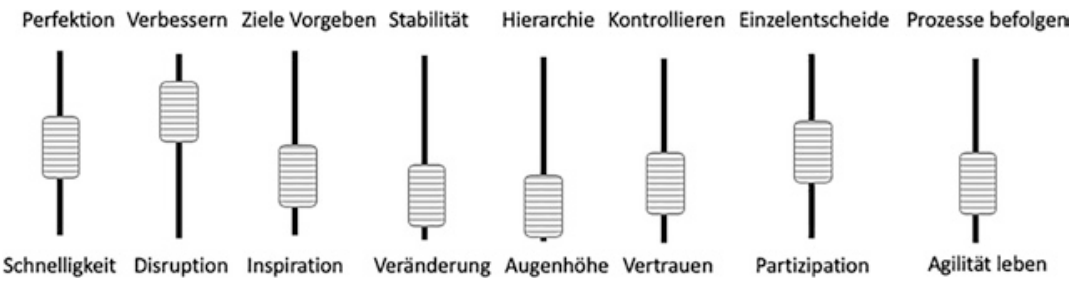

Abb. 5.2 Leitbild einer Führungskraft (vgl. Lindner 2019b) 
- Teambuilding: Auch virtuelle Teams müssen sich formen. Erste reale und punktuelle Treffen sind dabei förderlich.

- Konkrete Ziele: Es wird empfohlen, virtuelle Teams über Ziele zu steuern. Regelmäßige Statusmeldungen sind als Instrument zur Zielkorrektur sinnvoll.

- Klare Rollenverteilung: In einem virtuellen Team können eine stabile Rollenverteilung sowie das Arbeiten nach Pull-Prinzip eine Führungskraft entlasten.

- Mitarbeitern vertrauen: Virtuelle Führung basiert laut den Teilnehmern der Studie auf Vertrauen. Es kann davon ausgegangen werden, dass die delegierten Arbeitspakete mit steigendem Vertrauen umfangreicher werden.

- Basis: Es gibt das einheitliche Verständnis, dass eine Führungskraft nicht perfekt sein muss, Fehler zugeben darf oder einen ,schlechten Tag' haben kann.

- Primärcharakteristika sind Führung auf Augenhöhe, Vertrauen in Mitarbeiter, Experimente, Echtzeitinformationen und Inspiration fördern.

- Sekundärcharakteristika sind Echtzeitfeedback, Agilität vorleben, Partizipation und zur Veränderung motivieren.

- Neue Kompetenzen sind neue Trends antizipieren, agile Methoden und neue Arbeitskonzepte einsetzen, die Motivation der Mitarbeiter sowie Technologien evaluieren.

- Learning by Doing: Da Learning by Doing für Führungskräfte die am weitesten verbreitete Lernmethode darstellt, wird geraten, das Führungsleitbild direkt an den Arbeitsalltag anzupassen. Experimentierfreude ist gefragt!

Die ersten vier Empfehlungen fokussieren sich auf die eher operative Führung im direkten Umfang mit Mitarbeitern in virtuellen Teams. Sollte die COVID-19-Pandemie zurückgehen, wird es wieder möglich sein, persönliche Treffen abzuhalten. Diese sind auch bei virtuellen Teams wichtig und sollten immer eingeplant werden. Weiterhin sollten Sie nach konkreten Zielen vorgehen und Mitarbeitern stets Perspektiven aufzeigen.

Ein sehr wichtiger Punkt ist für mich die klare Rollenverteilung. Vermitteln Sie jedem Teammitglied, dass es wichtig für das Team ist und respektieren Sie die Rollen. Sagen Sie bei wichtigen Entscheidungen dazu: ,Wir können das nicht ohne Frau Müller entscheiden, da ihre Rolle die Qualitätssicherung ist. Sie muss an der Entscheidung beteiligt werden. ' So fördern Sie die Identifikation mit der Rolle und damit auch das Engagement der Mitarbeiter.

Sie werden feststellen, dass die weiteren Punkte eine Zusammenfassung des Leitbilds in Abb. 5.3 darstellen, die durch gewisse Kompetenzen erweitert werden. Führungskräfte werden dabei als sehr innovativ und motivierend 


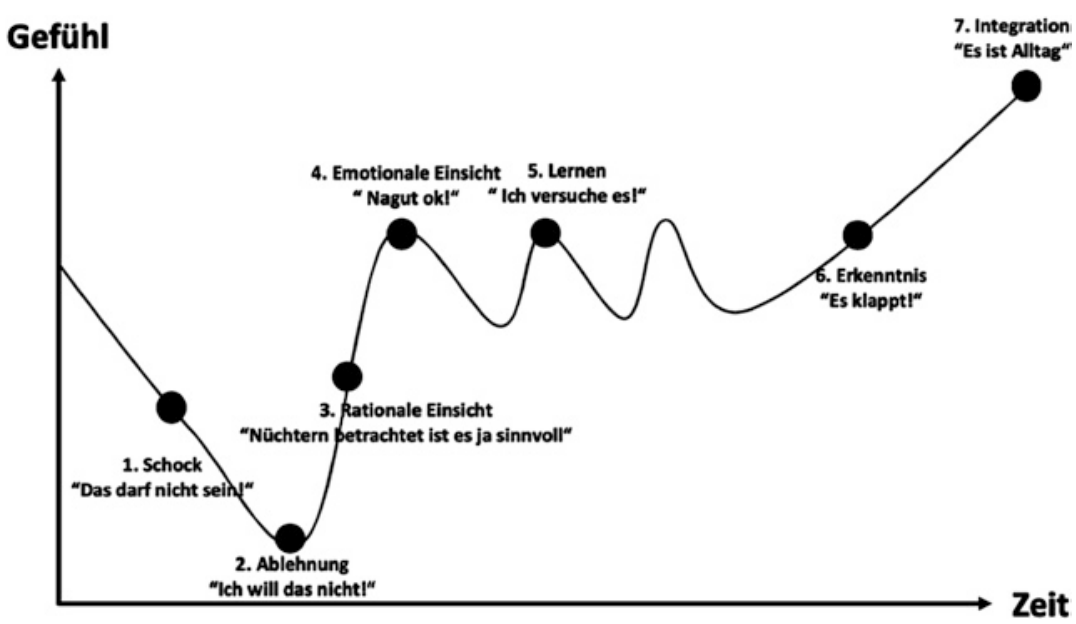

Abb. 5.3 Zyklus des Umgangs mit Veränderung nach Streich (2016) - eigene Abbildung

beschrieben. Doch wie lässt sich eine solche Änderung des Charakters umsetzen? Eine Möglichkeit ist laut den befragten Führungskräften das Learning bei Doing im Arbeitsalltag, das ich im folgenden Exkurs näher beschreiben möchte.

Merkbox Machen Sie sich immer bewusst, welche Bedeutung die One-on-one-Gespräche haben. Diese sind bei virtuellen Mitarbeitern besonders wichtig. Werden diese vernachlässigt, führt das zur Distanzierung von der Firma und zu einem Abfall der Motivation. Verschieben Sie diese Gespräche daher nur im Notfall.

\section{Exkurs: Änderung der eigenen Persönlichkeit durch Learning by Doing}

Nun stellt sich die Frage, ob eine Führungskraft einfach Gewohnheiten und Charakter ändern kann. Sehen Sie sich in der Lage, wie die jungen Startup-Gründer oder wie Jürgen Klopp oder Dieter Zetsche mit Ihrem Charakter ein Vorbild zu werden?

Natürlich können Menschen bewusst Änderungen in ihrer Persönlichkeit vornehmen, auch wenn dies ein gewisses Durchhaltevermögen erfordert. Jeder von uns hat tägliche Routinen (z. B. der Weg ins Büro, Zähneputzen vor dem Frühstück etc.) und Verhaltensweisen (z. B. emotionale Diskussion bei Problemen oder Rückzug bei starken Diskussionen). Hier sind die innersten und die tiefsten 
Routinen gemeint, die sich oft nur schwer beeinflussen lassen. Eine solche Veränderung besteht aus drei Schritten (vgl. Lindner 2019a):

- Schritt 1: Routinen erkennen

- Schritt 2: Motivationen erkennen

- Schritt 3: Neue Verhaltensweisen verinnerlichen

Als erstes sollten Sie Routinen erkennen, die Sie verändern oder sich aneignen möchten. Das lässt sich am besten mithilfe des Feedbacks von guten Freunden oder Arbeitskollegen erreichen. Solche Gewohnheiten können sein, dass Sie Mitarbeitern mehr zuhören wollen, weniger kontrollierend agieren und auch eine Geschichte aus dem Privatleben erzählen.

Das Verändern von Gewohnheiten ist ein Kann und kein Muss. Sie sollten also im nächsten Schritt eine Motivation zur Veränderung finden. Nicht jede Gewohnheit ist wichtig genug, um verändert zu werden, dazu muss nicht jeder Mensch perfekt sein. Fragen Sie sich: Ergeben sich negative Effekte durch eine bestimmte Verhaltensweise oder zeigen Manager in Ihren Umfeld Verhaltensweisen, die Sie bewundern?

Im dritten Schritt sollten Sie die neuen Verhaltensweisen im Alltag umsetzen. Das muss 66-mal geschehen, wobei dies mit einer kleinen Geste (z. B. einen Stück zuckerfreie Schokolade) belohnt werden sollte. Die Psychologin Philippa Lally vom University College in London hat dies erforscht, indem sie 96 Probanden die Aufgabe gab, sich drei neue Gewohnheiten (u. a. 15 min Spaziergang jeden Morgen) anzueignen. Die Anzahl der bewussten Ausführungen der Tätigkeit bis zur automatisierten Gewohnheit lag im Durchschnitt bei 66 (vgl. Lindner 2019a).

\subsection{Technologische Ebene: Teams auf Distanz steuern}

Neben der Sicherstellung der Motivation des Teams ist es notwendig, die Aufgabenerledigung im virtuellen Raum sicherzustellen. Hierzu sollten Sie Softwaretools zielführend einsetzen. Mir fällt oft auf, dass Führungskräfte versuchen, ein virtuelles Team mit herkömmlichen Methoden aus dem Büroalltag zu führen. Ein bekanntes Beispiel ist das Vertrauen auf den ,Flurfunk', durch den sich Informationen zwangsläufig verbreiten. Das funktioniert bei virtuellen Teams nicht, da diese räumlich verteilt sind und Informationen im Intranet, im Chat oder im täglichen Regelmeeting eigens kommuniziert werden müssen. 


\subsection{Empfehlung zur Führung auf technologischer Ebene}

Um sinnvolle Empfehlungen zur Nutzung von Technologie abzuleiten, habe ich die Führungskräfte aus der erwähnten Befragung um eine Bewertung der entsprechenden Software gebeten. Weiterhin sollten die Führungskräfte Informationen zu Vorgehen und Aufgabengröße geben. Mir war wichtig, das jeweilige Verhalten in Bezug auf die Software festzustellen. Die Tab. 5.1 fasst die Ergebnisse zusammen.

In der Tabelle werden verschiedene Software-Tools aufgeführt. In der ersten Spalte wird die Zufriedenheit der Führungskräfte mit den Software-Tools in der virtuellen Teamarbeit angegeben. $\mathrm{Zu}$ erkennen ist, dass vor allem die E-Mail in dieser Hinsicht eher abfällt und wahrscheinlich durch Chatsysteme und Telefon ersetzt wird.

Merkbox Effiziente virtuelle Teams sind laut meiner Erfahrung an einer geringen Anzahl der persönlichen (nicht automatisierten) E-Mails zu erkennen. Je besser die Kollaborationsinfrastruktur ist, desto mehr wird diese genutzt. Die E-Mail bleibt die Notlösung bspw. zur Kommunikation (Verteiler mit 15 Personen) und zur oftmals ineffizienten gemeinsamen Bearbeitung von Dokumenten (DokumentV3-final-final-final.docx). Auch privat werden kaum E-Mails mit Freunden ausgetauscht, sondern Instant-Messenger wie WhatsApp, Signal oder Telegram verwendet.

In der zweiten Spalte erfahren Sie mehr über das Vorgehen der Führungskräfte. Ich wollte wissen, ob das Software-Tool genutzt wird, um zu

Tab. 5.1 Ergebnisse der Studie von Lindner und Greff (2019) zur Aufgabenverteilung in virtuellen Teams

\begin{tabular}{l|l|l|l}
\hline Software & Zufriedenheit & Vorgehen & Aufgabengröße \\
\hline Jira & Hoch & Stark delegierend & Klein \\
\hline PM-Software & Hoch & Stark delegierend & Klein \\
\hline Videokonferenz & Sehr hoch & Delegierend & Groß \\
\hline Telefon & Hoch & Beides & Mittel \\
\hline E-Mail & Mittel & Kontrollierend & Klein \\
\hline Chatsystem & Sehr hoch & Kontrollierend & Klein \\
\hline
\end{tabular}


kontrollieren oder um Aufgaben schnell zu delegieren. Eine Empfehlung ist, dass Führungskräfte Aufgaben im Aufgabentool Jira einstellen und anschließend davon ausgehen können, dass diese erledigt wird. Meist wird dies im wöchentlichen Meeting geprüft oder es wird per E-Mail ein Status abgefragt.

Merkbox Abhängig vom Kanal und der virtuellen Kommunikation ist die passende Vorgehensweise zu wählen. E-Mail und Chat werden häufig zur Statusmeldung und zur Kontrolle genutzt. Videokonferenzen werden häufiger zur kreativen Entfaltung und als Vorgangsverwaltungstools zur Delegation verwendet.

Die dritte Spalte zeigt die Aufgabengröße. Während über Software wie Jira eher kleine Aufgaben vergeben werden, besprechen die Führungskräfte größere und mittlere Aufgaben via Videokonferenz oder per Telefon.

Merkbox Die Nutzung von Software zur Zusammenarbeit verändert die Arbeitsweise durch eine große Anzahl von Kleinstaufgaben, was deutlich schneller und dynamischer wirkt.

Ich selbst kann bestätigen, dass der Erfolg eines virtuellen Teams davon abhängig ist, wie es der Führungskraft gelingt, Arbeit im Software-Tool abzubilden und diese regelmäßig mit dem Team durchsprechen. Die Nutzung von Technologie zur Teamsteuerung schafft mehr Transparenz und eine höhere Dynamik.

Merkbox Für Führungskräfte fühlt es sich im ersten Schritt wie eine Degradierung zum Administrator einer Software an. Dabei wird deutlich mehr harte Führungsarbeit gefordert, als sich annehmen lässt, und Führungskräfte geraten an neue Grenzen.

Als ich 2019 Abteilungsleiter geworden bin, habe ich meine vier Teamleiter darin, weitergebildet, die virtuellen Teams durch Software konstruktiv zu steuern. Mittlerweile organisieren wir über diese Methodik weit über 50 Mitarbeiter. Die Arbeit verändert sich dadurch in einigen Punkten deutlich: Sie verwalten das Software-Tool als zentralen Informationsknoten und bestimmen auch Rechte (wer darf eine Aufgabe auf ,Fertig' setzen) und Prozesse (welche Phasen werden durchlaufen). Auch im Führungskräfte-Meeting schauen wir uns nur noch die Software-Tools gemeinsam an. Vorteile sind, dass Sie immer einen aktuellen Status für eventuelle Anfragen des Managements sowie eine Übersicht über Blocker (Störungen) im Team haben, die Sie als Manager beseitigen können. 


\subsection{Empfehlungen zum Veränderungsmanagement}

„Wenn der Wind der Veränderung weht, bauen die einen Mauern und die anderen Windmühlen“" (chinesisches Sprichwort).

Besonders die Einführung virtueller Teams sorgt für starke Veränderung und auch Ablehnung bei einigen Mitarbeitern. Jeder Mitarbeiter durchläuft bis zur Akzeptanz der Veränderung 7 Schritte. Einige Mitarbeiter durchlaufen diese in Sekunden und andere in Jahren. Der folgende Abschnitt gibt Hilfestellungen zu Veränderungen in Organisationen.

\section{Phase 1: Schock}

Die Notwendigkeit einer Veränderung erzeugt einen Schock durch Angst vor einer neuen Situation. Dies führt zu einer Unfähigkeit die Veränderung rational zu reflektieren. Meine Empfehlung in dieser Zeit: Kommunizieren Sie die Veränderung und lassen Sie die Mitarbeiter dann 1-2 Wochen in Ruhe den Schock verdauen. Sie verkünden lediglich, dass neue Softwaretools eingeführt werden, welche zu neuen Arbeitsmethoden führen.

\section{Phase 2: Ablehnung}

Nach dem Schock folgt eine starke Ablehnung. Sie bemerken diese Phase daran, dass Mitarbeiter anfangen über die Veränderung negativ zu reden. In dieser Phase sollten Sie als Führungskraft den Mitarbeitern helfen zu verstehen, warum die Änderung vorgenommen wird und damit Phase 3 vorbereiten. Erläutern Sie die Treiber für virtuelle Teams von Kap. 1 in diesem Essential verständlich für die Mitarbeiter. Sehen Sie sich als Anwalt der Veränderung.

\section{Phase 3: Rationale Einsicht}

Nachdem erkannt wird, dass Ablehnung gegen eine Veränderung nicht hilft, starten Mitarbeiter sich mit der Veränderung rational zu beschäftigen. Sie starten Vorteile abzuwägen, erste Informationen zur Veränderung aufzunehmen und wünschen sich erste kurzfristige Schritte zu deren Umsetzung. Kommunizieren Sie in dieser Phase erste Informationen zur Umsetzung der Veränderung jedoch nicht den gesamten Plan. Zeigen Sie beispielsweise erste Software-Tools und grundlegende Informationen zu agilen Methoden.

Merkbox Der Unterschied zwischen rationaler und emotionaler Einsicht kann sehr gut am Beispiel eins Fitnessstudios veranschaulicht werden. Ich sehe ein, dass ich zu dick bin aufgrund von Daten wie das Körpergewicht (rationale Einsicht) aber ich gehe nichts in Fitnessstudio, weil ich es nicht will (emotionale Einsicht). 


\section{Phase 4: Emotionale Einsicht}

Die emotionale Einsicht ist der eigentliche Wendepunkt und es heißt für Sie als Führungskraft dran zu bleiben. Mitarbeiter beginnen mit der Veränderung vertraut $\mathrm{zu}$ werden und zeigen sich offen. Wichtig sind in dieser Phase konkrete Projektpläne und erste Testzugriffe auf die Softwaretools zu gewähren.

\section{Phase 5: Lernen}

Die Mitarbeiter haben die Veränderung akzeptiert und starten die neuen Softwaretools und virtuelle Arbeitsmethoden zu erproben. Geben Sie erste Schulungen zu Software und Methoden sowie erste schriftliche Guidelines für die Mitarbeiter frei. Lassen Sie auch erste Teams mit den Software-Tools produktiv arbeiten.

\section{Phase 6: Erkenntnis}

Haben die Mitarbeiter in Phase 5 schnelle Erfolge mit den Software-Tools erzielt, werden diese sehr schnell die Veränderungen schrittweise in ihren Alltag übernehmen wollen. Stellen Sie einen netten Administrator zur Hilfestellung mit der Softwarenutzung zur Verfügung und bitten Sie um Feedback zu neuen Prozessen durch einen Prozessmanager.

\section{Phase 7: Integration}

Nun werden die neuen Software-Tools und Arbeitsweise als selbstverständlich angesehen. Ihre Aufgabe als Führungskraft ist es nun wie eine Art „Oberlehrer“ zu überwachen, ob die neuen Arbeitsweisen auch nach 2-3 Wochen noch eingehalten werden und Rückfälle in alte Verhaltensweisen vermieden werden.

\subsection{Fazit}

Besonders in den letzten Jahren hat eine neue Art des Führungsverhaltens die Management-Welt geprägt und sogar ein Fußballtrainer wurde Manager des Jahres. Es gilt daher, das eigene Verhalten zu hinterfragen und sich mit dem Charakter als Führungskraft auseinanderzusetzen. Der zweite Teil des Führungsverhaltens umfasst die konstruktive Nutzung von Technologie.

Ich habe in diesem Kapitel Empfehlungen zur Führung virtueller Teams gegeben. Dabei werden Sie festgestellt haben, dass sich die Führung virtueller Teams signifikant von der realer Teams unterscheidet. Es bedarf also einer Auseinandersetzung mit neuen Führungsmodellen, einer Reflexion der eigenen Führungsrolle und der konstruktiven Nutzung von Technologie. 
Der Weg zur virtuellen Führungskraft ist nicht einfach und sicher sind nicht alle Empfehlungen aktuell einfach umsetzbar. Ich habe ein virtuelles Team viele Manager in unterschiedlichen Altersstufen begleitet und kennengelernt, die umfassend an technologischen, aber auch an persönlichen Kompetenzen gearbeitet haben. All diese Manager erzählten mir, dass der Weg nicht leicht gewesen sei, aber zu einem großen Erfolg geführt habe. Auch ich versuche, jeden Tag meine Verhaltensweisen zu reflektieren und den Umgang mit technologischen Neuheiten zu erlernen, um in meiner noch recht jungen Führungskarriere weiterzukommen und dem Team ein Vorbild zu sein. 